\title{
A Reaction-Diffusion Model for Epidemic Routing in Sparsely Connected MANETs
}

\author{
Daniel J. Klein, João Hespanha, and Upamanyu Madhow \\ Dept. of Electrical \& Computer Eng. \\ University of California \\ Santa Barbara, CA 93106 \\ \{djklein, hespanha, madhow\}@ece.ucsb.edu
}

\begin{abstract}
We propose and investigate a deterministic traveling wave model for the progress of epidemic routing in disconnected mobile ad hoc networks. In epidemic routing, broadcast or unicast is achieved by exploiting mobility: message-carrying nodes "infect" non message-carrying nodes when they come within communication range of them. Early probabilistic analyses of epidemic routing follow a "well-mixed" model which ignores the spatial distribution of the infected nodes, and hence do not provide good performance estimates unless the node density is very low. More recent work has pointed out that the infection exhibits wave-like characteristics, but does not provide a detailed model of the wave propagation. In this paper, we model message propagation using a reaction-diffusion partial differential equation that has a traveling wave solution, and show that the performance predictions made by the model closely match simulations in regimes where the well-mixed model breaks down. In particular, we show that well-mixed models are generally overly optimistic in regard to the scaling of the message delivery delay with problem parameters such as communication range, node density, and total area. In contrast to prior work, our model provides insight into the spatial distribution of the "infection," and reveals that the performance is sensitive to the geometry of the deployment region, not just its area.
\end{abstract}

\section{INTRODUCTION}

We consider the problem of message dissemination in disconnected mobile ad hoc networks (MANETs). Such delaytolerant networks arise naturally in many contexts, including battlefield communication networks, animal tag based sensor networks [1], [2], and emergency response networks. In this setting, a given source node may be unable to reach its intended destination using multiple hops of communication for the current network topology. Instead, knowledge of the message spreads like an infection through the nodes as they employ short-range broadcast opportunistically; hence the commonly used term epidemic routing. Eventual delivery of the message is guaranteed for typical mobility models, so the main focus is on understanding how performance metrics such as end-to-end delay and memory requirements scale as a function of parameters such as the number of nodes, the communication range, the mobility model, and the geometry of the region over which the network is deployed. To this end, we derive a partial differential equation (PDE) model for the dissemination of information via epidemic routing.

Early analyses of epidemic routing employ a mathematical simplification that applies for exceedingly sparse deployments
[2], [3], [4], [5], [6]. In those settings, nodes meet sufficiently infrequently so that the infected, or informed, agents may be modeled as uniformly distributed in space at any given point of time, in which case it suffices to track only the number of informed agents. Following parlance common in the modeling of chemical reactions [7] (which in turn is similar to the modeling of the spread of infection), we use the term "wellmixed" for this regime, since spatial correlations wash away. While analytical predictions based on the well-mixed model exhibit remarkable agreement with simulations for very sparse deployments, they become increasingly inaccurate as the node density increases.

More recent work indicates that information dissemination via epidemic routing exhibits wave-like behavior. Kong and Yeh [8] use percolation theory to show that the delivery delay scales linearly with the source-destination distance when the network is in a subcritical (non-percolated) regime, consistent with wave-like propagation. Jacquet et al. [9], [10] explicitly point out wave-like propagation of information, using probabilistic journey analysis to derive upper bounds on the asymptotic speed of information propagation, and lower bounds on the asymptotic broadcast delay. These results are derived in an asymptotic regime, as the number of nodes and area of a square region tend to infinity at constant or diminishing node density. In contrast, our goal is to derive an explicit model for wave-like propagation that provides an accurate scaling law for the expected delivery delay in nonasymptotic regimes (and not necessarily square deployment regions).

Figure 1 shows simulation snapshots for information propagation in two regimes. The well-mixed model provides a good approximation in the very low-density regime (a), while radial wave-like behavior is evident in the higher density regime (b). To model the second regime, we obtain a PDE model of the spatiotemporal density of informed nodes that is closely related to models for population dispersal, chemical reactions, and the spread of infection.

Contributions: Our contributions are summarized as follows:

- We derive a reaction-diffusion PDE model for the propagation of information in MANETs using epidemic routing.

- We obtain traveling wave solutions to the PDE model 


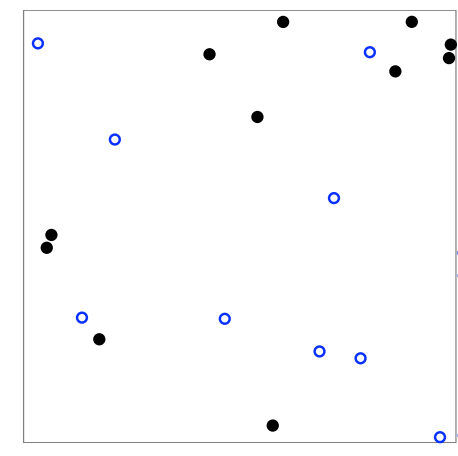

(a) Uniform Spread

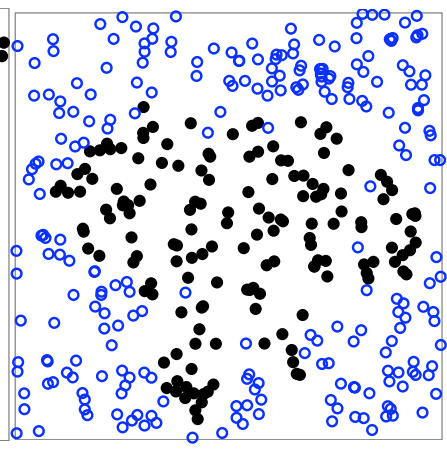

(b) Radial Wave Spread
Fig. 1: The above simulation snapshots show (a) a very sparse deployment in which informed nodes, represented by filled circles, are distributed essentially uniformly, and (b) a denser deployment exhibiting a wave of information expanding from a centrally located source. Existing well-mixed scaling laws apply to the situation in (a), whereas the reaction-diffusionbased laws derived in this paper apply to (b).

using techniques developed for spatial epidemics.

- We derive scaling laws expressing how the message delivery delay depends on the communication radius, node density, total area, and the region's shape.

- We show that there is a clear transition from the wellmixed regime to the wave propagation regime as the node density and/or the communication radius increases.

- We show that the geometry of the deployment region significantly affects information dissemination by studying rectangular regions with different aspect ratios. Such effects cannot be captured by the well-mixed model, since they require a detailed model for the spatio-temporal evolution of the "infection."

Related work: We have already mentioned prior models for epidemic routing, including well-mixed approximations [2], [3], [4], [5], [6] and probabilistic analyses indicating wave-like behavior [8], [9], [10]. As far as we know, the present paper is the first attempt to use PDEs to provide a detailed model of the wave-like spatiotemporal evolution of information propagation due to epidemic routing. In doing this, we are building on a long history of using PDEs to model similar phenomena such as population dispersal and spatially evolving epidemics. R. A. Fisher studied in 1937 a 1-D population undergoing spatial diffusion with a densitydependent Pearl-Verhulst (logistic) growth term [11], and traveling wave solutions were formalized the following year by Kolmogoroff, Petrovsky, and Piscounoff [12]. Extensions to 2D growth can be found in the 1951 work of Skellam [13]. In epidemics, slightly more complicated PDEs are used to model the spread of disease. The additional complexity comes from considerations for incubation periods, recovery, vaccination, and node death. The book by Rass and Radcliffe provides a good review of this work [14]. The MANET application differs from these prior models because we have a clear notion of the destination node, and are interested in studying message delay.

We note that the scaling laws for delay that we are interested in are different from the throughput scaling laws that have been the subject of intensive investigation since the seminal work of Gupta and Kumar [15], including work by Grossglauser and Tse [16] that shows that mobility can be exploited to improve throughput scaling. In contrast to the throughput scaling considered in these papers, our work, as well as the prior work in epidemic routing that we have mentioned above, focuses on delay scaling in disconnected MANETs.

Outline: The remainder of this paper is organized as follows. In Section II, the store-carry-and-copy routing protocol is described, and the well-mixed scaling law is reviewed. A reaction-diffusion partial differential equation model is introduced in Section III. A traveling wave solution to the PDE model is derived in Section IV, and resulting scaling laws, are described in Section V. In Section VI, the validity of the PDE model is tested in a simulation study of nodes employing a random direction mobility model. Concluding remarks and future directions are given in Section VII.

\section{Problem Formulation and Existing Results}

\section{A. Problem Formulation}

The routing problem is set in a large planar region $\mathcal{R}$ of total area $A$. Within this region, a total of $N$ nodes move randomly and independently according to a given mobility model. Consider a single source node that carries a message to be delivered to a particular destination node. Each node is able to communicate with any other node that wanders within a communication range $R$. Interference with other communications need not be considered because mobility operates at a timescale much slower than that of communication.

While the source could simply wait for the destination to wander within communication range, the end-to-end delay can be significantly reduced using a store-carry-and-copy protocol in which each node copies messages to other nodes that come within the communication range $R$ [17]. A figure of merit often used to describe the message passing process is the mean delivery delay (MDD), which is the expected elapsed time between the generation of the message at the source, and the final delivery of the message to the destination. The focus of this paper is on determining how this figure of merit scales with the problem data:

$\begin{array}{ll}\mathcal{R} & \text { A planar deployment region } \\ A & \text { Total area of } \mathcal{R} \\ V & \text { Node velocity } \\ N & \text { Number of nodes deployed } \\ R & \text { Communication radius }\end{array}$

Generally speaking, one would expect the delivery delay to decrease as $R, N$, or $V$ increase. Similarly, the delivery delay can be decreased by modifying the shape of the region $\mathcal{R}$ so that the average separation distance between the source and destination is reduced. The PDE model developed later applies to the general class of mobility models that can 


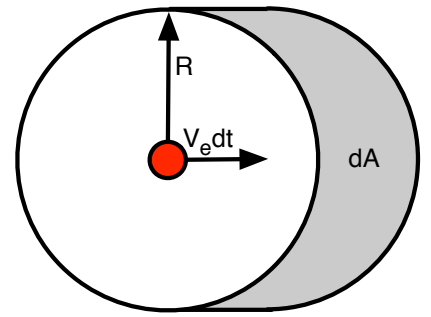

Fig. 2: The shaded region represents the area swept by the communication ball of an informed node with communication radius $R$ moving at relative speed $V_{e}$ for time $d t$.

be parameterized by a diffusion-like coefficient $D$ and an effective relative speed between nodes $V_{e}$. In the simulation section, we use the random direction mobility model.

\section{B. Existing Scaling Laws}

Scaling laws are a central focus of active research on mobility-assisted communication. Many authors have derived scaling laws of message delay for various protocols and parameters. In this paper, we focus on an opportunistic, time-optimal, routing protocol in which new information is forwarded every time two nodes meet. Previous scaling laws for this and other protocols are applicable only in "well-mixed" situations for which the node inter-meeting times are long compared to the mixing time of the mobility model. The mixing time of the system is a sufficiently large scalar $T_{\text {mix }}$ so that if two nodes start at the same position, their positions are essentially independent and uniformly distributed after an interval of length $T_{\text {mix }}$.

To pinpoint when the existing well-mixed laws are applicable, let $I(t)$ denote the expected number of informed nodes (i.e. those knowing the message) at time $t$ and assume that the nodes' density and communication radius are sufficiently small so that the time between consecutive encounters is larger than the mixing time, with large probability. After such time, the distribution of informed and uninformed nodes will each be uniform, with densities $I / A$ and $(N-I) / A$, respectively. As depicted in Fig. 2, in a small interval of time $d t$, the communication ball of each informed node sweeps a region of area

$$
d A=2 R V_{e} d t .
$$

Therefore, during this period, an average of $2 R V_{e}(N-I) / A d t$ uninformed nodes will be encountered, which means that the rate at which one informed node will meet uninformed nodes is equal to $2 R V_{e}(N-I) / A$. Assuming that all $I$ informed nodes encounter uninformed nodes at this rate, the expected number of nodes informed about a particular message can be described by the following ordinary differential equation,

$$
\frac{d I}{d t}=\frac{2 R V_{e}}{A} I(N-I)
$$

To determine under what conditions the well-mixed approximation is valid, note that the maximum expected rate

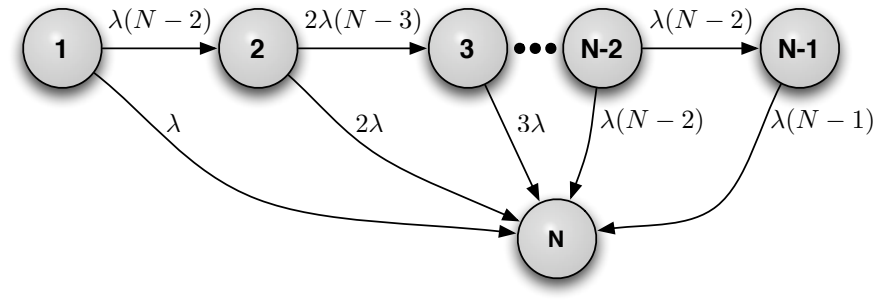

Fig. 3: The Markov model used in [3] to derive the well-mixed scaling law. The state represents the number of informed nodes, except for the destination node which is labeled as "N."

of message passing under a well-mixed assumption is

$$
\max _{I \in[0, N]} \frac{2 R V_{e} I(N-I)}{A}=\frac{R V_{e} N^{2}}{2 A},
$$

and therefore the expected time between message passing events is of order no smaller than

$$
\frac{2 A}{R V_{e} N^{2}}=\frac{2}{R V_{e} \rho_{0}^{2} A},
$$

where $\rho_{0}:=N / A$ denotes the nodes' density. For the wellmixed assumption to hold, this time must be larger than the mixing time, which means that

$$
\frac{2}{R V_{e} \rho_{0}^{2} A} \geq T_{\text {mix }} \quad \Leftrightarrow \quad \frac{2}{R \rho_{0}^{2} A^{\frac{3}{2}}} \geq \frac{V T_{\text {mix }}}{\sqrt{A}} .
$$

In practice, this relationship will hold for $R, \rho_{0}$, and $A$ small. It is important to note from (3) that the well-mixed assumption will fail to hold when the area or number of nodes is scaled-up at constant node density. One would expect the dimensionless quantity $V_{e} T_{\text {mix }} / \sqrt{A}$ to remain constant as one varies $V_{e}$ and $A$ for the same basic motion model of the nodes.

The well-mixed scaling law of Groenevelt et al. [3] comes from a combination of the above derivation of the fact that the nodes meet at a rate $\lambda:=2 R V_{e} / A$, with a Markov model, see Fig. 3. The Markov chain can be solved to arrive at the scaling law for the mean delivery delay,

$$
\begin{aligned}
\mathrm{MDD} & =\frac{A}{2 R V_{e}(N-1)} \sum_{i=1}^{N-1} \frac{1}{i} \\
& =\frac{A}{2 R V_{e}(N-1)}\left(\log (N-1)+\gamma+\mathcal{O}\left(\frac{1}{N-1}\right)\right) .
\end{aligned}
$$

Here, $\gamma \approx 0.577$ is Euler's constant.

\section{PDE APPROACH}

To go beyond the infrequent meetings required for the wellmixed scaling law to hold, in this section we derive a reactiondiffusion partial differential equation that will be used in the following sections to derive a new scaling law. The PDE models the evolution of the density of informed nodes, and we show that it reduces to the usual well-mixed ODE (2) under a uniform density assumption. However, when nodes instead 
meet sufficiently frequently so as to render the uniform density assumption invalid, traveling waves occur in the density of informed nodes because nodes initially close to the source will receive the message before those initially far from the source. The message passing process is thus spatial, and must be treated with a PDE instead of an ODE.

To begin, let $\rho_{I}(x, t)$ and $\rho_{U}(x, t)$ be the density of informed and uninformed nodes, respectively, at time $t$ and point $x \in \mathcal{R}$. Assume that the motion model admits a uniform steady state total density of nodes, $\rho_{0}:=N / A$, and that the nodes are initially spatially distributed uniformly. At any time and point we have

$$
\rho_{U}(x, t)+\rho_{I}(x, t)=\rho_{0} .
$$

Two factors cause the individual densities to change. The first is node mobility, which we approximate by diffusion. The time-rate of change of the density of informed nodes under diffusion alone varies as

$$
\begin{aligned}
\frac{\partial \rho_{I}(x, t)}{\partial t} & =D\left(\frac{\partial^{2}}{\partial x_{1}^{2}}+\frac{\partial^{2}}{\partial x_{2}^{2}}\right) \rho_{I}(x, t) \\
& =D \Delta \rho_{I}(x, t),
\end{aligned}
$$

where $D$ is the effective diffusion coefficient of the motion model with units of distance ${ }^{2} /$ time from Fick's laws [18], and $\Delta$ is the Laplace operator.

For a continuous time random walk mobility model, the effective diffusion coefficient is just the usual diffusion coefficient computed as

$$
D=\frac{1}{2 n} \frac{E\left[\|x(t+d t)-x(t)\|^{2}\right]}{d t}, \forall t, d t
$$

where $n=2$ is the dimension of the walk. For other mobility models, like the random direction model used in the simulation section, the ratio in (9) may not be constant for every $d t>0$, but it may still be approximately constant for the relevant time intervals. For such models, the constant $D$ in (7) should be viewed as an effective diffusion coefficient that is specific to the motion model under consideration and that can be estimated through Monte Carlo simulations or sometimes derived theoretically [19]. Once $D$ is determined, it can be used as $N$ and $R$ are varied.

The second factor influencing density is the copying of message from one node to another. As this process unfolds, $\rho_{I}(x, t)$ increases locally and $\rho_{U}(x, t)$ decreases so that the steady state density is preserved. The rate at which $\rho_{U}(x, t)$ is converted to $\rho_{I}(x, t)$ locally is determined by the flux of message knowledge across the communication boundary of radius $R$ about each uninformed node. As discussed in Section II, in a small interval of time of length $d t$, an informed node at a point $x$ sweeps a small area $2 R V_{e} d t$ given by (1). In this area, one would expect to find $2 R V_{e} \rho_{U}(x, t) d t$ uninformed nodes. If we now consider the effect of all informed nodes in a small area $d A$ centered at $x$, we have $\rho_{I}(x, t) d A$ informed nodes, each passing messages to uninformed nodes at rate $2 R V_{e} \rho_{U}(x, t)$. This leads to a rate of infection per unit of time and per unit of area equal to

$$
2 R V_{e} \rho_{I}(x, t) \rho_{U}(x, t),
$$

resulting in a time-rate of change of $\rho_{I}(x, t)$ given by

$$
\frac{\partial \rho_{I}(x, t)}{\partial t}=2 R V_{e} \rho_{I}(x, t) \rho_{U}(x, t),
$$

which using (6) becomes

$$
\frac{\partial \rho_{I}(x, t)}{\partial t}=2 R V_{e} \rho_{I}(x, t)\left(\frac{N}{A}-\rho_{I}(x, t)\right) .
$$

It is interesting to note that this equation is equivalent to a logistic growth model known as the Pearl-Verhulst model in spatial ecology [20]. The Pearl-Verhulst model has the form,

$$
\frac{\partial \rho(x, t)}{\partial t}=r_{o} \rho(x, t)\left(1-\frac{\rho(x, t)}{K}\right),
$$

where $r_{o}$ is the intrinsic growth rate and $K$ is the carrying capacity. From (12), we find an intrinsic growth rate of $r_{o}=2 R V_{e} N / A$ and carrying capacity of $K=N / A$. The Pearl-Verhulst model gives a "reproduction" rate for each node that depends on the local population density, instead of growing at a constant rate as in the Malthusian (exponential) growth model. In the MANET context, this density dependent growth is credible since each informed node is most effective at passing messages when surrounded mostly by uninformed nodes, because when other informed nodes are nearby, competition for uninformed nodes makes each informed node pass messages (reproduce) at a lower rate. The local message passing rate is zero at the carrying capacity $\rho_{I}(x, t)=N / A$ because all nearby nodes have already received the message.

Under the combined diffusion (7) and message passing (12), the net time-rate of change of the density of informed nodes is given by

$$
\frac{\partial \rho_{I}(x, t)}{\partial t}=D \Delta \rho_{I}(x, t)+2 R V_{e} \rho_{I}(x, t)\left(\frac{N}{A}-\rho_{I}(x, t)\right),
$$

which is a two-dimensional version of a classic nonlinear reaction-diffusion equation known as the Fisher-KolmogoroffPetrovsky-Piscounoff (FKPP) equation [21].

As one might expect, the well-mixed ODE for the expected number of informed nodes (2) can be recovered from the reaction-diffusion PDE (14). Specifically, the expected number of informed nodes,

$$
I(t)=\int_{\mathcal{R}} \rho_{I}(x, t) d x
$$

varies as

$$
\begin{aligned}
\frac{d I(t)}{d t} & =\int_{\mathcal{R}} \frac{\partial \rho_{I}(x, t)}{\partial t} d x \\
& =\int_{\mathcal{R}} D \Delta \rho_{I}(x, t)+2 R V_{e} \rho_{I}(x, t)\left(\frac{N}{A}-\rho_{I}(x, t)\right) d x \\
& =2 R V_{e} \int_{\mathcal{R}} \rho_{I}(x, t)\left(\frac{N}{A}-\rho_{I}(x, t)\right) d x
\end{aligned}
$$


where the final equality follows from the fact that diffusion only spreads nodes around and does not increase the number of informed nodes. If we then assume that the density is uniform so that $\rho_{I}(x, t)=I(t) / A$ for all $x$, we find that (18) reduces to

$$
\frac{d I}{d t}=\frac{2 R V_{e}}{A} I(N-I),
$$

which is precisely the well-mixed model (2).

In modeling the speed at which information propagates from the source $x_{s}$, it is convenient to study the radial component $r=\left\|x-x_{s}\right\|$ of the polar form of the FKPP equation (14),

$$
\begin{aligned}
\frac{\partial \rho_{I}(r, t)}{\partial t}=D\left(\frac{\partial^{2}}{\partial r^{2}}\right. & \left.+\frac{1}{r} \frac{\partial}{\partial r}\right) \rho_{I}(r, t) \\
& +2 R V_{e} \rho_{I}(r, t)\left(\frac{N}{A}-\rho_{I}(r, t)\right) .
\end{aligned}
$$

For $r$ sufficiently large, (20) reduces to the one-dimensional FKPP equation,

$$
\frac{\partial \rho_{I}(r, t)}{\partial t}=D \frac{\partial^{2} \rho_{I}(r, t)}{\partial r^{2}}+2 R V_{e} \rho_{I}(r, t)\left(\frac{N}{A}-\rho_{I}(r, t)\right) .
$$

Remark 1: The derivation of the infection rate in (10) presumed a motion model in which nodes move with constant velocity over short time intervals (see Fig 2). While this is reasonable for most motion models, including the widely used random direction model considered in Section VI, it would technically not be true, e.g., for a pure random walk for which the paths are discontinuous at every point. However, even in this case, our model would still apply because all that we need is a linear relationship between the area increment swept by a node in a small time interval of length $d t$ (the region depicted in gray in Fig. 2) and the product $2 R d t$ as in (1). This type of relation would still hold for motion models like a random walk, except that the area increment would have a different shape than the one in Fig. 2 (it would essentially be an annulus) and $V_{e}$ should be understood as an effective velocity defined as the ratio between the area increment and $2 R d t$. By the same token, a circular communication range is also not crucial for this model, as different shapes would still result in a similar relationship between the area increment and the time interval of length $d t$.

\section{Traveling Wave Solution}

Partial differential equations can be difficult to solve in general. One approach frequently taken is to look for a traveling waveform solution. That is to say we look for a solution to $\rho_{I}(r, t)$ of the form,

$$
\rho_{I}(r, t)=\Omega(r-c t),
$$

for some waveform $\Omega: \mathbb{R} \rightarrow \mathbb{R}^{+}$and wave speed $c$ [21]. The one-dimensional FKPP equation (21) is well-known to have a traveling wave solution, which we derive for the MANET

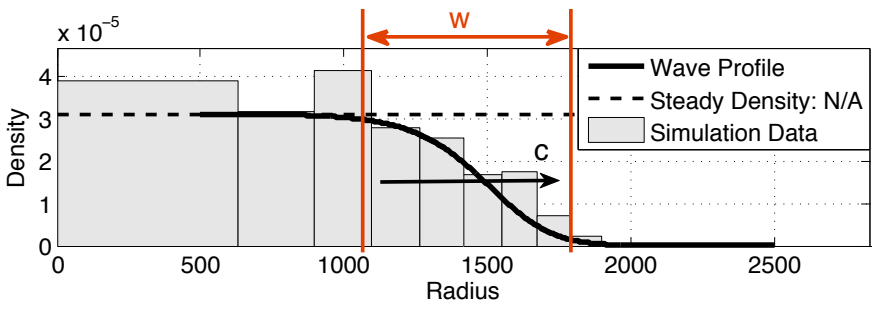

Fig. 4: A traveling wave profile (24) derived from the reactiondiffusion partial differential equation is drawn over simulation data from Section VI. The waveform travels to the right at speed $c$ and has width $w$ as indicated.

problem in Appendix A. The result is that a traveling wave solution exists for a wave speed of

$$
c=2 \sqrt{\frac{2 R V_{e} N D}{A}} .
$$

No closed form solution is known for the waveform $\Omega$, but a good approximation from [21] reveals the density of informed agents to be

$$
\rho_{I}(r, t)=\frac{N}{A}\left[\frac{r_{0} D e^{r_{0} z / c}}{c^{2}\left(1+e^{r_{0} z / c}\right)^{2}} \log \left(\frac{4 e^{r_{0} z / c}}{\left(1+e^{r_{0} z / c}\right)^{2}}\right)+\right.
$$

neglecting terms of order $c^{-4}$ and higher, with

$$
r_{0}=\frac{2 R V_{e} N}{A}, \quad z=r-c t .
$$

A waveform and example simulation data for the MANET parameters considered in Section VI is shown in Fig. 4.

Remark 2: We note that the traveling wave approach to solving the PDE (14) does not respect the fact that nodes cannot leave the region, and thus is not always appropriate. Nonetheless, we expect the wave solution to be accurate when the wave width, see Fig. 4, is less than the size of the region,

$$
w=8 \sqrt{\frac{2 D}{\rho_{0} R V_{e}}}<\sqrt{A} .
$$

\section{SCALING LAW}

To derive the scaling laws that follow, we divide the expected source-destination distance by the wave speed computed in Section IV:

$$
\begin{aligned}
\mathrm{MDD} & =\frac{1}{c} E\left[\left\|x_{s}-x_{d}\right\|\right] \\
& =\sqrt{\frac{A}{8 R V_{e} N D}} E\left[\left\|x_{s}-x_{d}\right\|\right] .
\end{aligned}
$$

For motion models with a uniform steady-state distribution and expected net motion equal to zero, such as a random walk or a random heading model, the expected distance between the source and destination should be computed as the distance 
between two points selected uniformly randomly [10]. For rectangular regions with side lengths of $a$ and $b$, this expected distance is [22],

$$
\begin{aligned}
E\left[\| x_{s}-\right. & \left.x_{d} \|\right]=\frac{1}{3} a b 2+\frac{a^{2}}{6 b} \log \left(\frac{b+a b 2}{a}\right) \\
+ & \frac{b^{2}}{6 a} \log \left(\frac{a+a b 2}{b}\right)-\frac{a b 2^{5}}{15 a^{2} b^{2}}+\frac{a^{5}+b^{5}}{15 a^{2} b^{2}},
\end{aligned}
$$

where $a b 2=\sqrt{a^{2}+b^{2}}$, which when the region is a square with $a=b$ simplifies to

$$
E\left[\left\|x_{s}-x_{d}\right\|\right]=\frac{a}{15}(2+\sqrt{2}+5 \log (1+\sqrt{2})) \approx 0.5214 a .
$$

From (28) and (30), we conclude that the overall scaling law for the message delivery delay in a square region of side length $a=\sqrt{A}$ and random source and destination is given by

$$
\mathrm{MDD}_{\text {square }}=\kappa \frac{A}{\sqrt{2 R V_{e} D N}},
$$

where

$$
\kappa=\frac{1}{30}(2+\sqrt{2}+5 \log (1+\sqrt{2})) \approx 0.1776 .
$$

The above results for the mean delivery delay in a non-wellmixed system should be compared to the well-mixed model (5).

A few key observation stem from this work.

1) The MDD scales with $1 / \sqrt{R}$ instead of the $1 / R$, which would be predicted by the well-mixed model. This means that doubling the communication radius only decreases the MDD by $30 \%$, instead of $50 \%$.

2) The MDD scales with $A / \sqrt{N}=\sqrt{A / \rho_{0}}$, instead of $A \log (N) / N=\log \left(A \rho_{0}\right) / \rho_{0}$, which would be predicted by the well-mixed model. This means that if we double the node density while keeping the area fixed, the MDD is only decreased by $30 \%$, instead of $50 \%$. Alternatively, if we double the area, while keeping the node density fixed, the MDD increases by $41 \%$, instead of a small additive increase, which would be predicted by the wellmixed model (simply due to the $\log \left(A \rho_{0}\right)$ term).

Overal, we conclude that the previously derived well-mixed models are generally overly optimistic in regard to the scaling of the MDD with the problem parameters. While some of the changes in scaling are simple percentage adjustments (e.g., $30 \%$ vs. $50 \%$ ), the scaling law with the total area is fundamentally different, as the well-mixed model predicts essentially no growth of delay with area, whereas under reasonable network parameters the delay is likely to grow with the square-root of the area. In fact, for different geometries (28) may lead to even worse scaling scaling behaviors.

\section{Vi. Simulation Results}

In this section, we present simulation results to support the PDE-based traveling wave scaling law. We begin by describing the node mobility model and simulation environment from which the data are generated. Then, we show an example
TABLE I: Default parameters used in simulations.

\begin{tabular}{|c|c|c|}
\hline Parameter & Meaning & Value \\
\hline \hline$N$ & Number of nodes & 200 \\
\hline$A$ & Area & $16 \mathrm{~km}^{2}$ \\
\hline$\gamma$ & Rectangular region aspect ratio & 1 \\
\hline$R$ & Communication radius & $100 \mathrm{~m}$ \\
\hline$V$ & Absolute node speed & $1 \mathrm{~m} / \mathrm{s}$ \\
\hline$V_{e}$ & Effective relative speed & $1.27 \mathrm{~m} / \mathrm{s}$ \\
\hline$\tau$ & Mean time between heading changes & $900 \mathrm{~s}$ \\
\hline$D$ & Effective diffusion coefficient & $28 \mathrm{~m}^{2} / \mathrm{s}$ \\
\hline
\end{tabular}

in which the traveling wave solution accurately predicts the spatial spread of informed nodes in a rectangular region. Finally, we compare the well-mixed (5) and PDE (28) scaling laws through parametric studies of how the delivery delay scales with the number of nodes, communication radius, and aspect ratio of a rectangular region.

\section{A. Node Mobility Model and Simulation Environment}

Each node follows a random direction mobility model in which periods of linear motion are interrupted by random heading changes [23]. Specifically, each node travels straight at constant speed $V$ for an exponentially distributed amount of time with mean $\tau$ before choosing a new heading uniformly on $[0,2 \pi)$. Nodes are reflected off any region boundaries that they may encounter. For the large values of $\tau$ used in the simulations below, heading changes are infrequent so the effective node relative velocity is essentially the average relative speed between two nodes,

$$
V_{e}=\frac{1}{2 \pi} \int_{0}^{2 \pi} \sqrt{(V+V \cos (\theta))^{2}+(V \sin (\theta))^{2}} d \theta=\frac{4 V}{\pi} .
$$

Parameter values used throughout this section, unless stated otherwise, are given in Table I. These parameter values reflect problems of interest to the U.S. Army [24]. The effective diffusion coefficient was determined through Monte Carlo simulations for the specific motion model considered here, and was not varied throughout trials. However, it should be emphasized that the specific value of this coefficient, as well as the value of $V_{e}$ above, does not affect the scaling laws, as they simply shift vertically the log-log plots in Figs. 6 and 7. Simulation trials were conducted from random initial conditions in a custom $\mathrm{C}++$ application.

\section{B. Traveling Wave Propagation}

We first demonstrate the correspondence between the traveling wave solution for the density of informed agents and simulation data. In Fig. 5, the position of informed (filled) and uninformed (empty) nodes are shown at several points in time. The red arc is centered at the initial location of the source and has radius $c t$, with wave speed $c$ computed using (23). Data for this figure was generated with $N=250$ nodes, an aspect ratio of $\gamma=3$, and all other parameter values given in Table I. 


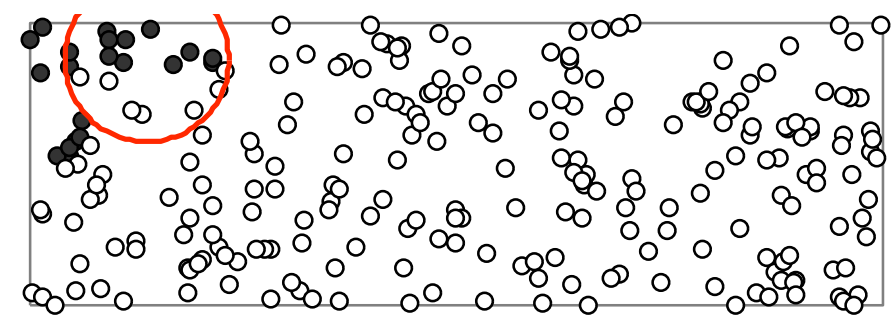

(a) $\mathrm{t}=1000 \mathrm{~s}$

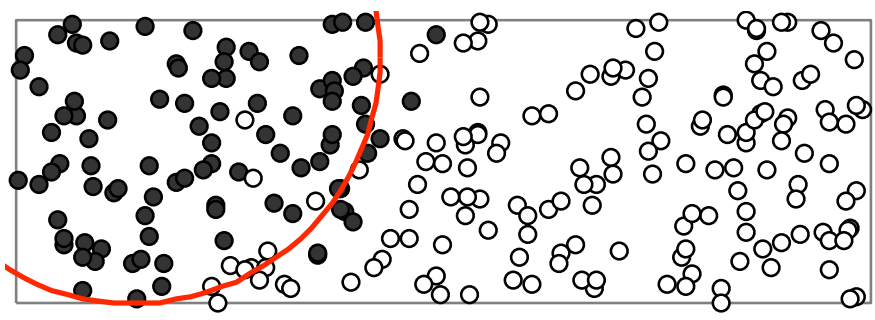

(b) $t=3000 \mathrm{~s}$

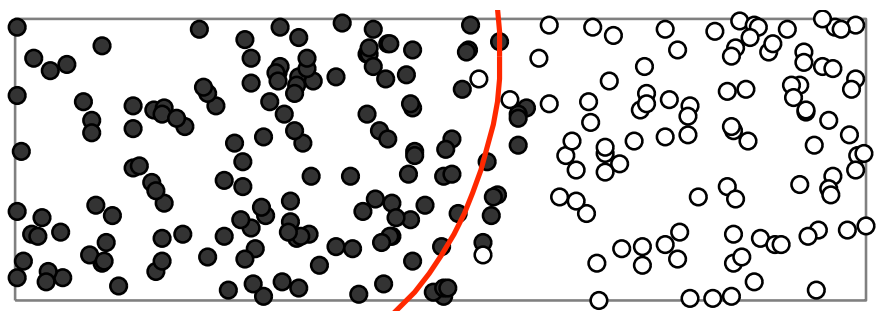

(c) $\mathrm{t}=4500 \mathrm{~s}$

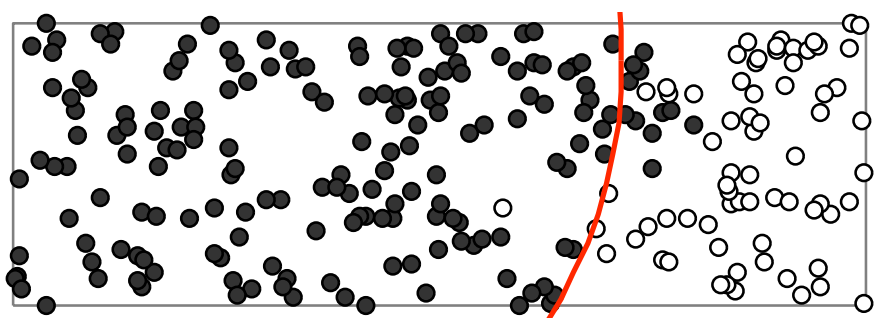

(d) $\mathrm{t}=6000 \mathrm{~s}$

Fig. 5: The location of informed (filled) and uninformed (empty) nodes is shown at several points in time to demonstrate the traveling wave solution. The leading edge of the wave is represented by the red arc, which is centered on the initial position of the source and has radius $c t$.

In addition to the leading edge of the traveling wave closely matching simulation data, the analytical solution for the density (24) also well approximates the informed node density. Fig. 4 from Section IV showed the traveling waveform over simulation data generated with $N=500$ agents, a centrally located source, and all other parameters as given in Table I.

\section{Scaling Results}

Number of Nodes: In this first simulation study, we vary the number of nodes, while keeping all other parameters at the values in Table I. The results in Fig. 6 indicate that the well-mixed model accurately describes the scaling law

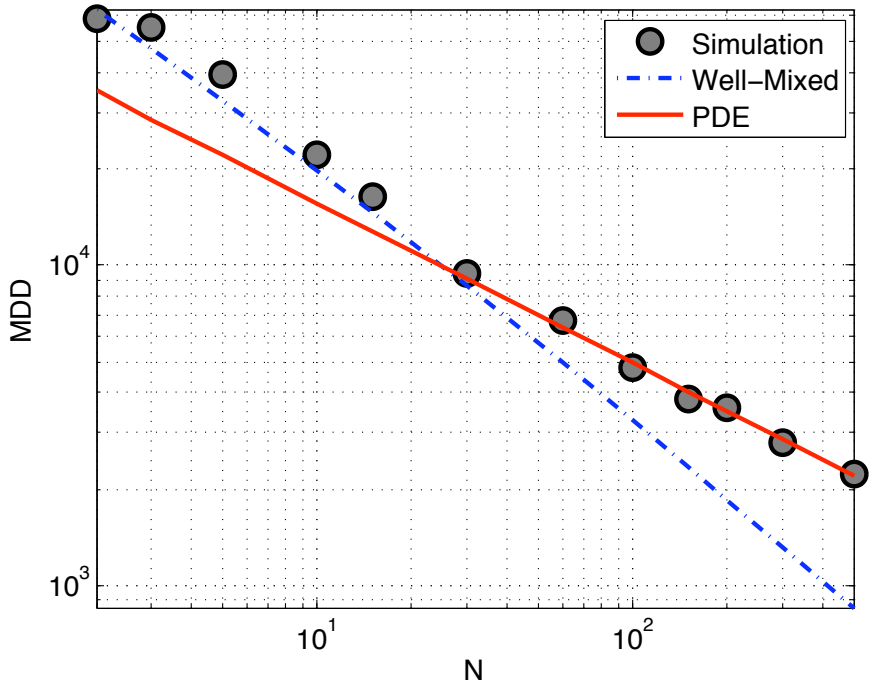

Fig. 6: Scaling the number of agents shows a transition from well-mixed to wave behavior. For the parameters used here, this transition takes place near $N=25$ nodes.

at low node densities $\left(N / A<25\right.$ nodes $\left./ 16 \mathrm{~km}^{2}\right)$ for which the system is well-mixed. The traveling wave solution is not appropriate in this regime because the wave is wider than the region (26). As the number of nodes increases, the well-mixed assumption (3) breaks down while the wave width decreases so that the traveling wave solution becomes accurate. The expected delivery delay scales as $\log (N) / N$ according to the well-mixed model, and $1 / \sqrt{N}$ according to the traveling wave model.

Both scaling laws suggest that increasing the number of agents should decrease the mean delivery delay, a trend clearly exhibited in the data. The difference in scaling comes from the fact that the well-mixed model inherently assumes that each informed node is equally efficient at spreading the message, whereas the wave model takes into account the fact that only nodes at the front of the wave are effective. As $N$ becomes large the node density increases and only a fraction of the nodes (i.e. those at the front of the wave) are effective, so the well-mixed model gives predictions that are too optimistic (smaller delays).

Communication Radius: In this second simulation study, we vary the communication radius keeping all other parameters at their default values. The results are shown in Fig. 7. Again, a transition from well-mixed to traveling wave behavior is apparent, this time the traveling wave accurately predicts the simulation results for communication radii above $R=30 \mathrm{~m}$. The expected delivery delay scales as $1 / R$ for the well-mixed model, in contrast to the $1 / \sqrt{R}$ scaling for the traveling wave model.

When $R$ is small for a given region, the well-mixed assumption (3) holds. Nodes that are initially close are likely to pass by each other without communicating, resulting in a process that unfolds approximately uniformly throughout the area. The 


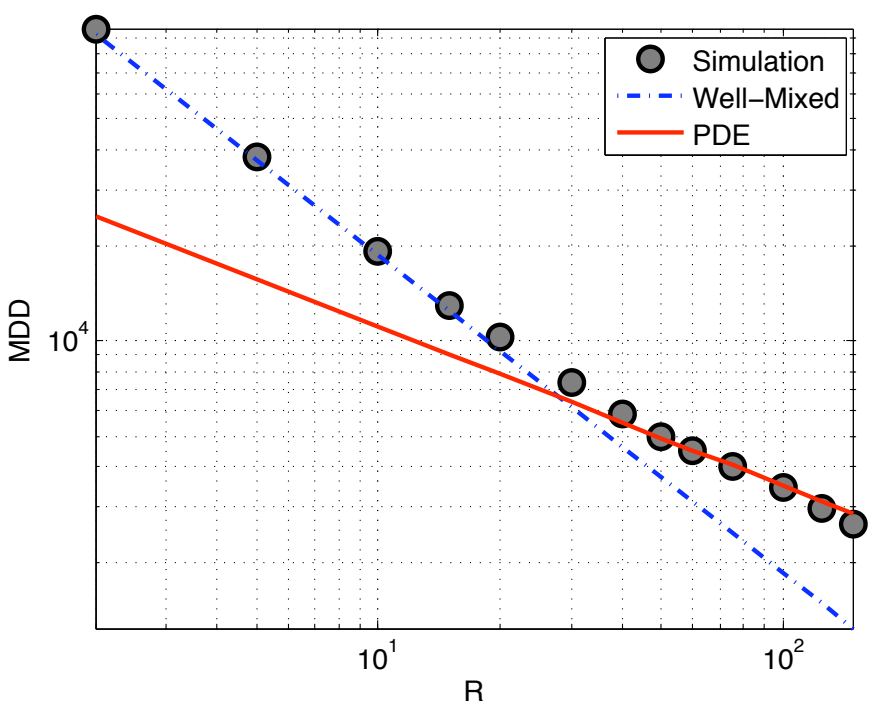

Fig. 7: Scaling of the mean delivery delay with communication radius $R$. A transition from well-mixed to PDE wave behavior is apparent near $R=30 \mathrm{~m}$.

traveling wave solution is not appropriate in this regime as the wave is too wide for the size of the region (26). As the communication radius is increased, the well-mixed assumption breaks down while the wave width decreases to a point where the traveling wave solution becomes accurate. A given node will then almost surely meet nearby nodes before meeting those initially far away, an effect which triggers the traveling wave behavior modeled by the PDE. Note that the transition from well-mixed to traveling wave would happen at $R$ less than $30 \mathrm{~m}$ if the problem was scaled up in area or number of nodes at constant node density.

Geometry: Finally, we explore scaling of the delivery delay with the geometry of the deployment region. Here, geometry is quantified by the aspect ratio (i.e. length divided by width) of a rectangular region with the nominal area of $16 \mathrm{~km}^{2}$. For this experiment, $N=250$ nodes were used for which traveling waves are triggered even for an aspect ratio of one. Fig. 8 shows the predictions of mean delivery delay as a function of the aspect ratio for the well-mixed and traveling wave models. The estimates from the well-mixed model depend only on the total deployment area, and therefore cannot account for the significant impact of aspect ratio on performance. This leads to an overestimation of the number of informed agents in the proximity of uninformed agents. The estimated mean delivery time is overly optimistic, reaching an error of $83 \%$ as the aspect ratio increases. In contrast, the traveling wave model provides accurate estimates, with prediction error reaching only $11 \%$ even at large aspect ratios. This is because the mean delivery delay for the traveling model is estimated as the expected source-destination distance divided by the wave speed, and the former increases according to (29) with aspect ratio.

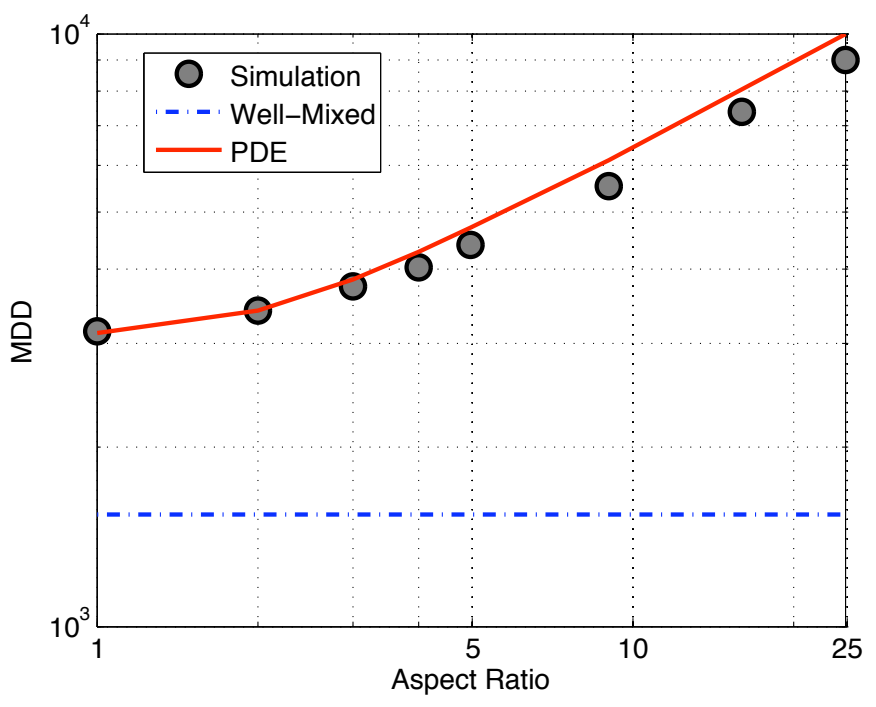

Fig. 8: Scaling with the aspect ratio of a rectangular region of constant area. The traveling wave model gives mean delivery delay predictions that are a function of the aspect ratio whereas the well-mixed model does not.

\section{CONCLUSION}

The results of this paper show that the complex, stochastic, spatiotemporal evolution of epidemic routing is well approximated by a deterministic reaction-diffusion model for information propagation. Our model provides accurate performance estimates for moderate node densities, with information dissemination occurring as a traveling wave propagating out from the information source. Since the well-mixed model suggested in prior analyses of epidemic routing provides accurate estimates when the density evolves uniformly, a combination of the two models is applicable over a broad range of parameter values. Note, however, that traveling waves cannot be avoided as the area or number of nodes is scaled up at constant density. Unlike the well-mixed model, the reaction-diffusion model captures the effect of the geometry of the deployment region on information propagation. For rectangular deployment regions, the reaction-diffusion model gives reasonably good predictions over a large range of aspect ratios.

An important topic for future work is to evaluate our approach for a variety of mobility models, and determine how the effective diffusion coefficient $D$ and the effective relative velocity $V_{e}$ depend on the mobility model. We would also like to model the effect of minor protocol variations (e.g., message deletion upon timer expiry to reduce node memory requirements) on the delivery delay. From a system design viewpoint, it is important to extend the model to a mix of agents with widely different mobility characteristics. This would enable us to determine, for example, if a small number of highly mobile agents can drastically speed up information propagation. 


\section{APPENDIX A}

\section{Derivation of the Traveling WaVE SpeED}

The key observation needed to solve the traveling wave solution is that the partial differential equation for $\rho_{I}(r, t)$ becomes an ordinary differential equation for $\Omega(z)$, where $z=r-c t$. Substituting the waveform (22) into the onedimensional FKPP equation (21) reveals,

$$
-c \frac{d \Omega(z)}{d z}=D \frac{d^{2} \Omega(z)}{d z^{2}}+2 R V_{e} \Omega(z)\left(\frac{N}{A}-\Omega(z)\right),
$$

a second order nonlinear differential equation for $\Omega(z)$. Letting $\Psi(z)=d \Omega(z) / d z$, (34) can be written as

$$
\frac{d}{d z}\left[\begin{array}{l}
\Omega \\
\Psi
\end{array}\right]=\left[\begin{array}{c}
\Psi \\
-\frac{c}{D} \Psi-\frac{2 R V_{e}}{D} \Omega\left(\frac{N}{A}-\Omega\right)
\end{array}\right] .
$$

A closed form solution to this equation is not available in general. However, we are looking for a particular solution for $\Omega(z)$ that tends to $N / A$ as $z \rightarrow-\infty$ and to 0 as $z \rightarrow \infty$. In other words, we are looking for a solution that has all nodes informed well behind the wavefront, and none of the nodes informed well ahead of the wavefront. The equilibria of (35) are $(\Omega(z), \Psi(z))=\{(0,0),(N / A, 0)\}$, so the desired solution must go from the $(N / A, 0)$ equilibrium point to the $(0,0)$ equilibrium point. Such a solution, connecting one equilibrium point to another, is called a heteroclinic orbit.

A further constraint on the solution for the waveform is that the density of informed nodes cannot be negative, so $\Omega(z) \geq 0$ for all $z$. To determine if a non-negative solution can exist for the waveform, we first linearize (35),

$$
\frac{d}{d z}\left[\begin{array}{l}
\Omega \\
\Psi
\end{array}\right]=\left[\begin{array}{cc}
0 & 1 \\
-\frac{2 R V_{e} N}{A D} & \frac{4 R V_{e} \Omega-c}{D}
\end{array}\right]\left[\begin{array}{l}
\Omega \\
\Psi
\end{array}\right] .
$$

At $(\Omega, \Psi)=(0,0)$, the eigenvalues of the linearization are

$$
\lambda_{ \pm}=-\frac{c}{D} \pm \sqrt{\frac{c^{2}}{D^{2}}-\frac{8 R V_{e} N}{A D}}
$$

which have negative real part indicating that this equilibrium point is stable, as desired. However, to ensure that the waveform $\Omega(z)$ remains non-negative, the solution cannot oscillate about the origin. To ensure no oscillation, we must have both eigenvalues real, so

$$
c \geq 2 \sqrt{\frac{2 R V_{e} N D}{A}} .
$$

A range of wave speeds are possible, and each wave speed gives a slightly different wave profile. However, most initial conditions result in a wave that translates at the minimum speed, so we take equality in (38) [12]. Unlike the onedimensional PDE (21), the two-dimensional PDE (20) does not have traveling wave solutions at small distances from the source due to the additional $\frac{D}{r} \frac{\partial \rho_{I}}{\partial r}$ term. However, one can see that the speed of information propagation at small $r$ will be slightly slower because the density $\rho_{I}$ decreases with increasing radius $r$ making $\frac{\partial \rho_{I}}{\partial r}$ negative.

\section{ACKNOWLEDGMENT}

This material is based upon work supported by the Institute for Collaborative Biotechnologies through grant DAAD19-03D-0004 from the U.S. Army Research Office.

\section{REFERENCES}

[1] P. Juang, H. Oki, Y. Wang, M. Martonosi, L. Peh, and D. Rubenstein, "Energy-efficient computing for wildlife tracking: Design tradeoffs and early experiences with zebranet," COMPUTER ARCHITECTURE NEWS, vol. 30, no. 5, pp. 96-107, 2002.

[2] T. Small and Z. J. Haas, "The shared wireless infostation model: a new ad hoc networking paradigm (or where there is a whale, there is a way)," in MobiHoc '03: Proceedings of the 4th ACM international symposium on Mobile ad hoc networking \& computing. ACM, 2003, pp. 233-244.

[3] R. Groenevelt, P. Nain, and G. Koole, "The message delay in mobile ad hoc networks," Performance Evaluation, vol. 62, no. 1-4, pp. 210-228, 2005.

[4] X. Zhang, G. Neglia, J. Kurose, and D. Towsley, "Performance modeling of epidemic routing," Computer Networks, vol. 51, no. 10, pp. 2867$2891,2007$.

[5] A. Jindal and K. Psounis, "Proceedings of the 2006 international conference on wireless communications and mobile computing," Proceedings of the 2006 international conference on Wireless communications and mobile computing, Jan 2006.

[6] G. Sharma and R. Mazumdar, "Scaling laws for capacity and delay in wireless ad hoc networks with random mobility," 2004 IEEE International Conference on Communications, Jan 2004.

[7] D. Gillespie et al., "Exact stochastic simulation of coupled chemical reactions," The journal of physical chemistry, vol. 81, no. 25 , pp. 2340 2361, 1977.

[8] Z. Kong and E. Yeh, "On the latency for information dissemination in mobile wireless networks," in Proceedings of the 9th ACM international symposium on Mobile ad hoc networking and computing. ACM New York, NY, USA, 2008, pp. 139-148.

[9] P. Jacquet, B. Mans, and G. Rodolakis, "Information Propagation Speed in Mobile and Delay Tolerant Networks," INFOCOM, 2009.

[10] — - "Broadcast Delay of Epidemic Routing in Intermittently Connected Networks," in IEEE International Symposium on Information Theory, 2008. ISIT 2009, 2009.

[11] R. Fisher, "The wave of advance of advantageous genes," Ann. Eugenics, vol. 7, pp. 353-369, 1937.

[12] A. Kolmogoroff, I. Petrovsky, and N. Piscounoff, "Etude de l'équation de la diffusion avec croissance de la quantité de matière et son application à un problème biologique," Bull. Univ, Etat. Moscou Ser. Int. A, vol. 1, pp. 1-25, 1937.

[13] J. Skellam, "Random dispersal in theoretical populations," Biometrika, vol. 38, pp. 196-218.

[14] L. Rass and J. Radcliffe, Spatial deterministic epidemics. American Mathematical Society, 2003.

[15] P. Gupta and P. Kumar, "The capacity of wireless networks," IEEE Transactions on information theory, vol. 46, no. 2, pp. 388-404, 2000.

[16] M. Grossglauser and D. Tse, "Mobility increases the capacity of ad hoc wireless networks," IEEE/ACM Transactions on Networking (ToN), vol. 10 , no. 4 , pp. 477-486, 2002.

[17] A. Vahdat and D. Becker, "Epidemic routing for partially connected ad hoc networks," Technical Report CS-200006, Duke University, Tech. Rep., 2000.

[18] A. Fick, "Ueber diffusion," Annalen der physik und chemie, vol. 170, no. $1,1855$.

[19] G. Taylor, "Diffusion by continuous movements," Proc. London Math. Soc, vol. 20, no. 2, pp. 196-211, 1921.

[20] J. Murray, Mathematical biology. Springer Verlag, 2003.

[21] J. Logan and W. InterScience, An introduction to nonlinear partial differential equations. Wiley-Interscience, 2008.

[22] B. Gaboune, G. Laporte, and F. Soumis, "Expected distances between two uniformly distributed random points in rectangles and rectangular parallelpipeds," The Journal of the Operational Research Society, vol. 44, no. 5, pp. 513-519, 1993.

[23] C. Bettstetter, "Mobility modeling in wireless networks: categorization, smooth movement, and border effects," ACM SIGMOBILE Mobile Computing and Communications Review, vol. 5, no. 3, pp. 55-66, 2001.

[24] Swami, A., Personal communication, 2009. 